\title{
Size Dependence of the Nonlinear Elastic Softening of Nanoscale Graphene Monolayers under Plane-Strain Bulge Tests: A Molecular Dynamics Study
}

\author{
Sukky Jun, ${ }^{1}$ Tenzin Tashi, ${ }^{1}$ and Harold S. Park ${ }^{2}$ \\ ${ }^{1}$ Department of Mechanical Engineering, University of Wyoming, Laramie, WY 82071, USA \\ ${ }^{2}$ Department of Mechanical Engineering, Boston University, Boston, MA 02215, USA
}

Correspondence should be addressed to Sukky Jun, sjun@uwyo.edu

Received 28 May 2010; Accepted 19 October 2010

Academic Editor: Sulin Zhang

Copyright (C) 2011 Sukky Jun et al. This is an open access article distributed under the Creative Commons Attribution License, which permits unrestricted use, distribution, and reproduction in any medium, provided the original work is properly cited.

\begin{abstract}
The pressure bulge test is an experimental technique to characterize the mechanical properties of microscale thin films. Here, we perform constant-temperature molecular dynamics simulations of the plane-strain cylindrical bulge test of nanosized monolayer graphene subjected to high gas pressure induced by hydrogen molecules. We observe a nonlinear elastic softening of the graphene with an increase in hydrogen pressure due to the stretching and weakening of the carbon-carbon bonds; we further observe that this softening behavior depends upon the size of the graphene monolayers. Our simulation results suggest that the traditional microscale bulge formulas, which assume constant elastic moduli, should be modified to incorporate the size dependence and elastic softening that occur in nanosized graphene bulge tests.
\end{abstract}

\section{Introduction}

Along with the indentation hardness test, the pressure bulge test has been one of the popular experimental techniques to characterize the mechanical properties of microscale thin films [1]. Recently, Bunch et al. [2] applied the bulge test technique to a graphene monolayer, or a one-atomthick two-dimensional crystalline sheet of carbon atoms, in order to predict its elastic behavior, while Lee et al. [3] measured the elastic properties and intrinsic breaking strength of free-standing monolayer graphene membranes by nanoindentation in an atomic force microscope. Both experimental studies employed graphene membranes with dimensions on the order of a few microns. On the other hand, molecular dynamics (MD) simulations have often been employed for scales of length much smaller than micrometers, due to the intractable computational expense that would be incurred for a direct experimental comparison. There have been numerous examples of MD simulations of nanoindentation of a variety of nanometer materials [4-6]. However, to the authors' knowledge, no atomistic simulation of the pressure bulge test has been reported for the analysis of the nonlinear elastic properties of atomic layers, in particular nanometer-sized graphene monolayers, which motivates the current study. We therefore perform constanttemperature MD simulations of plane-strain bulge tests of graphene monolayers to investigate their nonlinear elastic response to extremely high pressures generated by hydrogen molecules.

\section{Simulation Method}

The schematic model of the atomistic plane-strain pressure bulge test that we perform in the present work is illustrated in Figure 1, where the graphene monolayer is represented by the gray half-cylindrical sheet. Before applying the pressure, the hollow chamber underneath the undeformed flat graphene monolayer has initial dimensions of $2 a \times d \times w$, where the cross sectional area is $2 a \times d$ and the longitudinal length along the axis of cylindrical symmetry, which is much longer than the other dimensions, is $w$. After a certain amount of pressure is applied by supplying hydrogen molecules $\left(\mathrm{H}_{2}\right)$ into the chamber, the graphene monolayer is inflated upward and eventually reaches a thermally equilibrated bulge height $h$. 


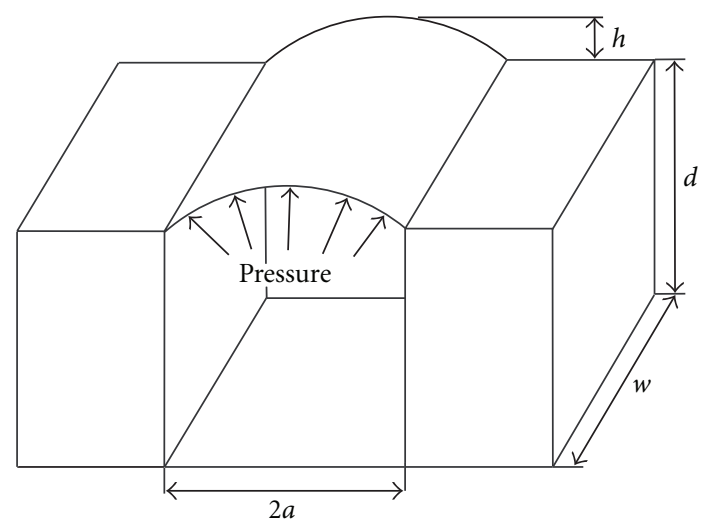

Figure 1: Schematic of the model employed to perform the cylindrical pressure bulge test.

Constant-temperature molecular dynamics simulations were performed by employing the Nosé-Hoover thermostat $[7,8]$, while the carbon-carbon interactions of the graphene monolayer are modeled using the hydrocarbon reactive empirical bond-order potential (known as AIREBO, i.e., Adaptive Intermolecular Reactive Empirical Bond-Order potential) [9] as implemented in the publicly available LAMMPS atomistic simulation package [10]. The simulation time step is fixed as $0.5 \mathrm{fs}$ and the temperature is set to be $300 \mathrm{~K}$. We employ a vertical slab model for the simulation box as shown in Figure 2.

Initially, we place an energy-minimized flat graphene monolayer in the simulation box and fix the positions of edge atoms closest to the two narrow side walls to represent idealized van der Waal's clamping to an underlying substrate. The slab width $w$ is relatively small compared with the other dimensions of the box because we impose periodic boundary conditions on the front and rear faces perpendicular to the $w$ direction to mimic an infinitely extended graphene monolayer. We impose reflecting boundary conditions on the other four-side faces [10]. While the space above the graphene monolayer is kept as vacuum, we supply sufficient hydrogen molecules into the space underneath the graphene monolayer such that the monolayer is inflated upward due to the hydrogen pressure, where the $\mathrm{C}-\mathrm{H}$ and $\mathrm{H}-\mathrm{H}$ interactions are also represented by the same above-mentioned AIREBO potential [9]. Hydrogen molecules were employed to induce the pressure mainly for the convenience of being able to utilize the same AIREBO interatomic potential for all $(\mathrm{C}-\mathrm{C}$, $\mathrm{C}-\mathrm{H}$, and $\mathrm{H}-\mathrm{H})$ molecular interactions.

After the graphene monolayer reaches the thermally equilibrated state, we measure the height of the bulge $h$. The new expanded volume underneath the graphene monolayer is calculated by simple geometrical analysis, assuming that the curve of the graphene is part of a perfect circle (i.e., pressure is homogeneously applied). The gas pressure is computed by contributions of both the kinetic energy and the virial energy, as implemented in LAMMPS [10], after excluding any contribution from the $\mathrm{C}-\mathrm{C}$ interactions within the graphene monolayer. We tested four graphene monolayers of different length, that is, $2 a=40,80,120$, and

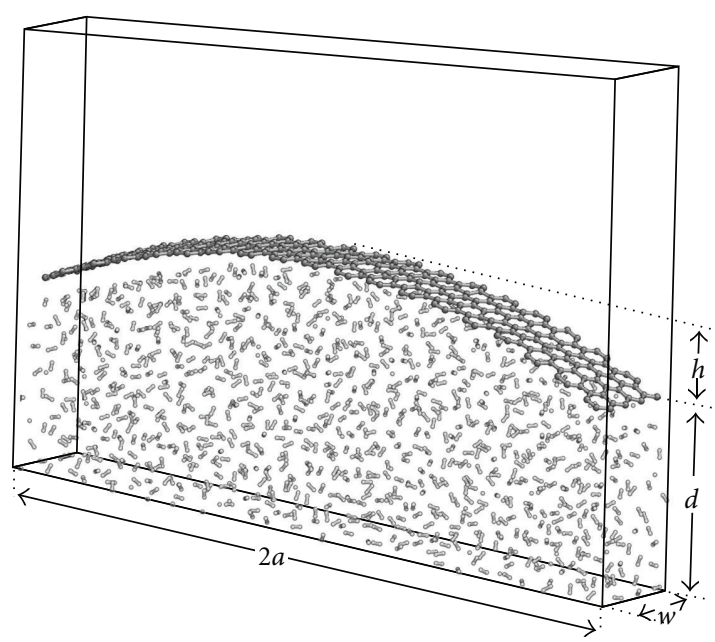

Figure 2: Atomistic simulation model of the nanoscale cylindrical bulge test of the graphene monolayer. The schematic shows the graphene monolayer bulged to height $h$ due to the pressure exerted by the $\mathrm{H}_{2}$ molecules underneath it.

$160 \AA$, where for each graphene monolayer of length $2 a$, the above procedure was repeated several times by changing the number of hydrogen molecules to change the pressure.

\section{Results and Discussion}

We first present the typical response of a graphene monolayer using the model with length $2 a=80 \AA$ and width $w=9.8 \AA$ that is composed of 300 carbon atoms. The entire simulation box is $80.4 \AA \times 80 \AA \times 10 \AA$ including the vacuum zone, and the graphene monolayer is initially placed at a height of $d=$ $20 \AA$ from the bottom wall, as shown in Figure 2. Figure 3(a) depicts the plot of the bulge height versus hydrogen pressure as the number of hydrogen molecules is increased. The corresponding pressure ranges from about 2407 to 5900 bar $(0.24 \sim 0.59 \mathrm{GPa})$, which are very high pressures, and leads to bulge heights ranging from $4.47 \AA$ to $21.20 \AA$ (it is noted that extreme pressures as high as $1.3 \mathrm{Mbar}$ have been reported by a recent first-principles study of fullerene nanocages filled with hydrogen molecules [11]). The fitted line in Figure 3(a) clearly exhibits softening behavior from early stage, which is in sharp contrast to the hardening obtained from the bulge test of microscale graphene [2]. Our simulation reveals that the nanosized graphene monolayer continues softening until a $\mathrm{C}-\mathrm{C}$ bond is broken at a pressure of about 6600 bar.

In order to demonstrate the discrepancy between our MD simulation results and classical elasticity more clearly, we derived the analytical elasticity solution for the relationship between pressure $p$ and bulge height $h$ of the cylindrical bulge test, considering large deflection of the graphene, as follows:

$$
p=\frac{C_{1}}{R}\left[\frac{R}{a} \arcsin \left(\frac{a}{R}\right)-1\right]+\frac{C_{2}}{R}\left[\frac{R}{a} \arcsin \left(\frac{a}{R}\right)-1\right]^{2},
$$




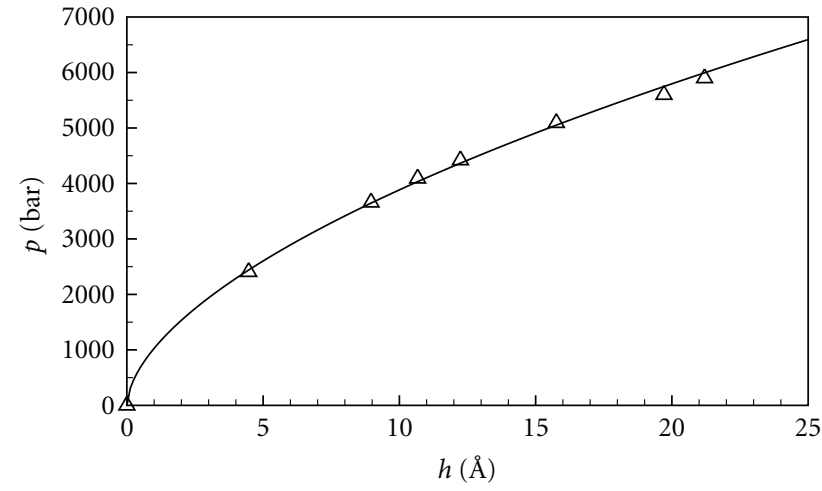

(a)

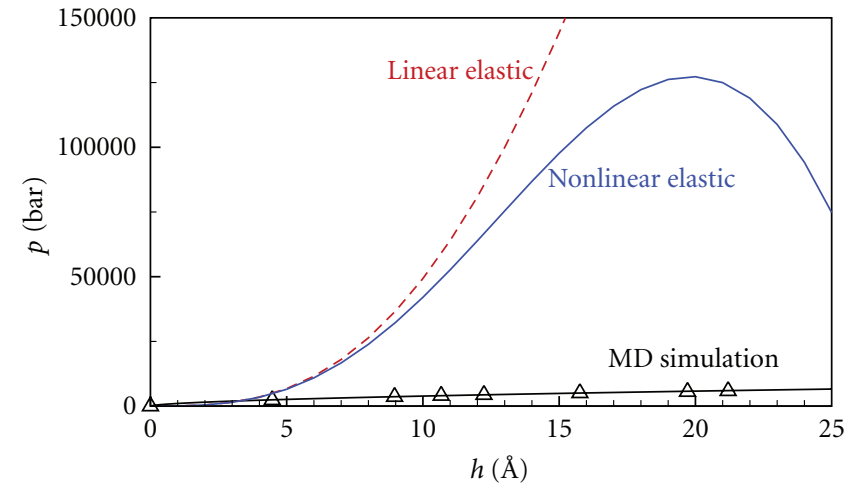

(b)

FIgURE 3: (a) The response of the $2 a=80 \AA$ graphene monolayer to pressure induced by hydrogen molecules. As the pressure increases, the graphene deflects more easily, indicating softening behavior. (b) MD results of (a) are compared with classical elasticity solutions for largedeflection bulge tests. Typical microscale linear elastic thin films follow $p \propto h^{3}$ curves. Nanoscale MD simulation reveals that the graphene monolayer is easier to deflect at much lower pressure than elasticity solutions.

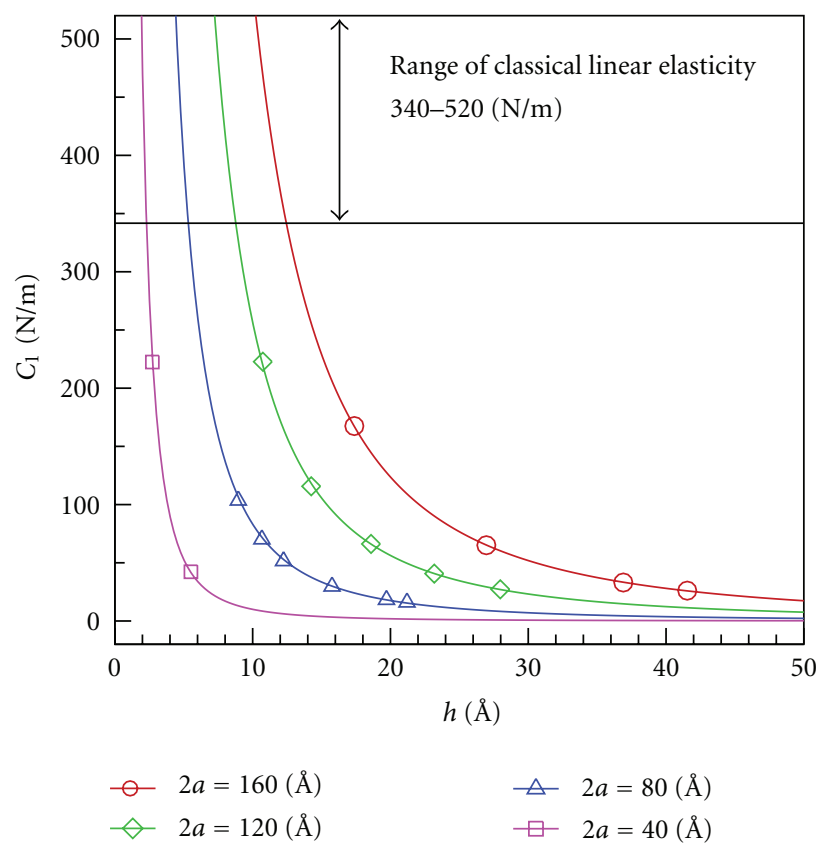

(a)

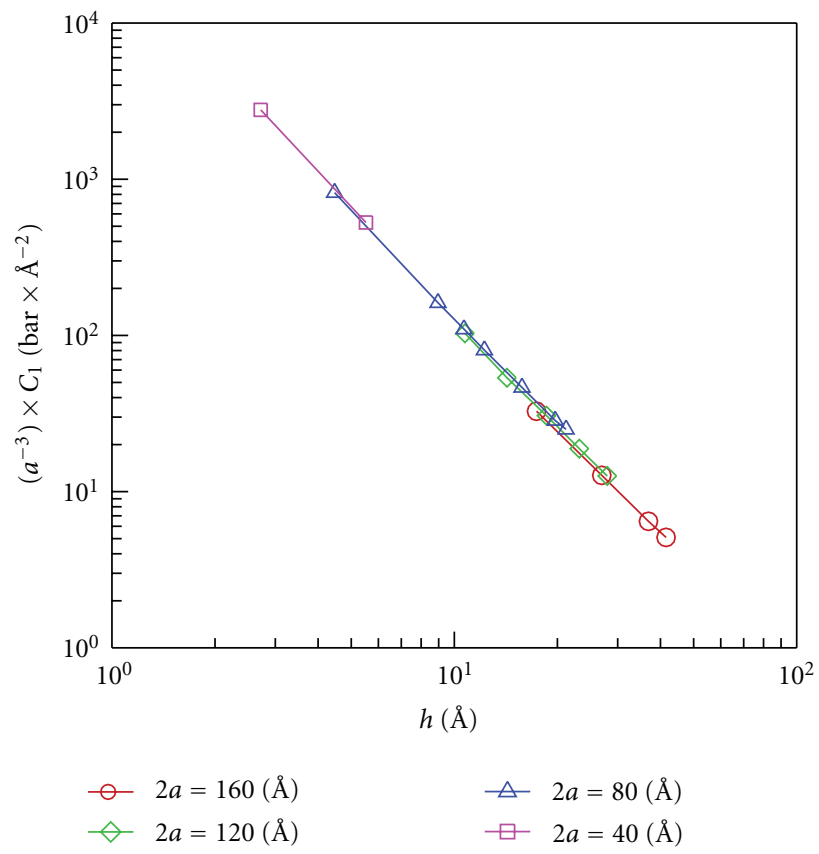

(b)

Figure 4: (color online) Elastic constant $C_{1}(\varepsilon)$ that is computed from the simulation data. (a) Illustration of the size-dependent softening behavior that is common amongst all four graphene monolayers under consideration. (b) Collapse of the size-dependent values of the elastic constant onto a single line on a log scale after multiplication by the size factor $a^{-3}$.

where $R=\left(a^{2}+h^{2}\right) / 2 h$. A simple material nonlinearity, $\sigma=C_{1} \varepsilon+C_{2} \varepsilon^{2}$, was assumed as employed in an experimental study of microscale graphene indentation [3] where authors deduced the values of $C_{1}=340(\mathrm{~N} / \mathrm{m})$ and $C_{2}=$ $-690(\mathrm{~N} / \mathrm{m})$. Using these values, we plot the elasticity solutions together with our MD results in Figure 3(b). Linear elastic curve implies $C_{2}=0$. It is well shown that our nanoscale graphene specimen is easier to deflect at much lower hydrogen pressure than elasticity solutions, which exhibits the softened response. We also simulated three other graphene monolayers of different lengths, that is, $2 a=40$, 120 , and $160 \AA$. Under similar pressure levels, we found this softening behavior to be common amongst all the simulated graphene monolayers.

In our $300 \mathrm{~K}$ molecular dynamics simulations, we did not measure the bulge height when the gas pressure was relatively low. This is because the graphene monolayer exhibits thermal fluctuations such that extracting an equilibrated bulge 


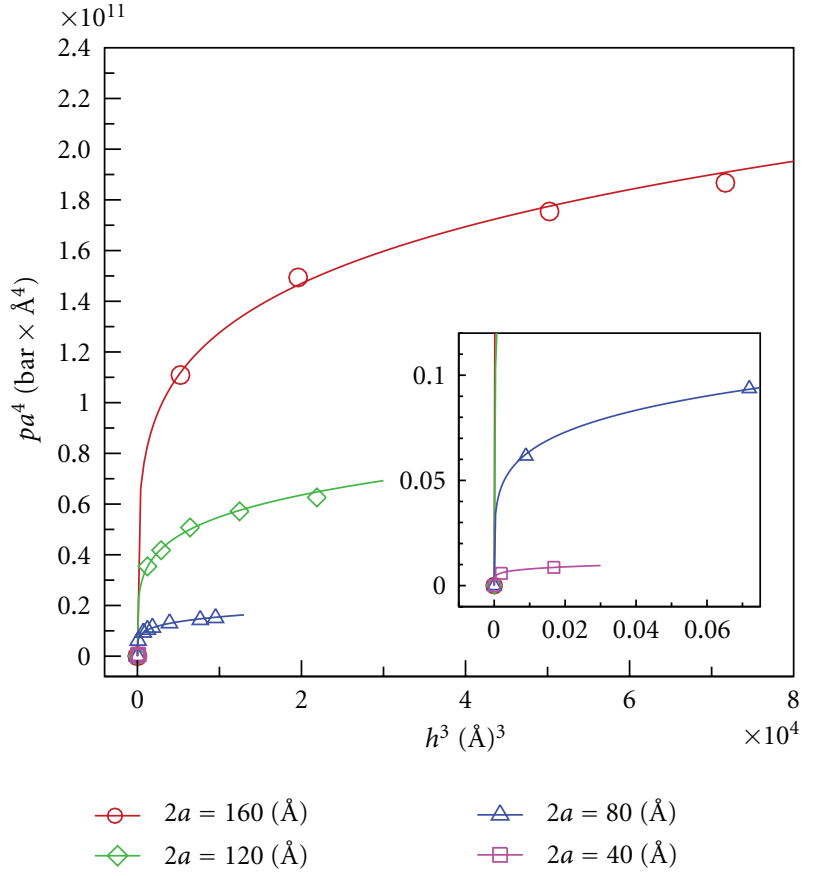

(a)

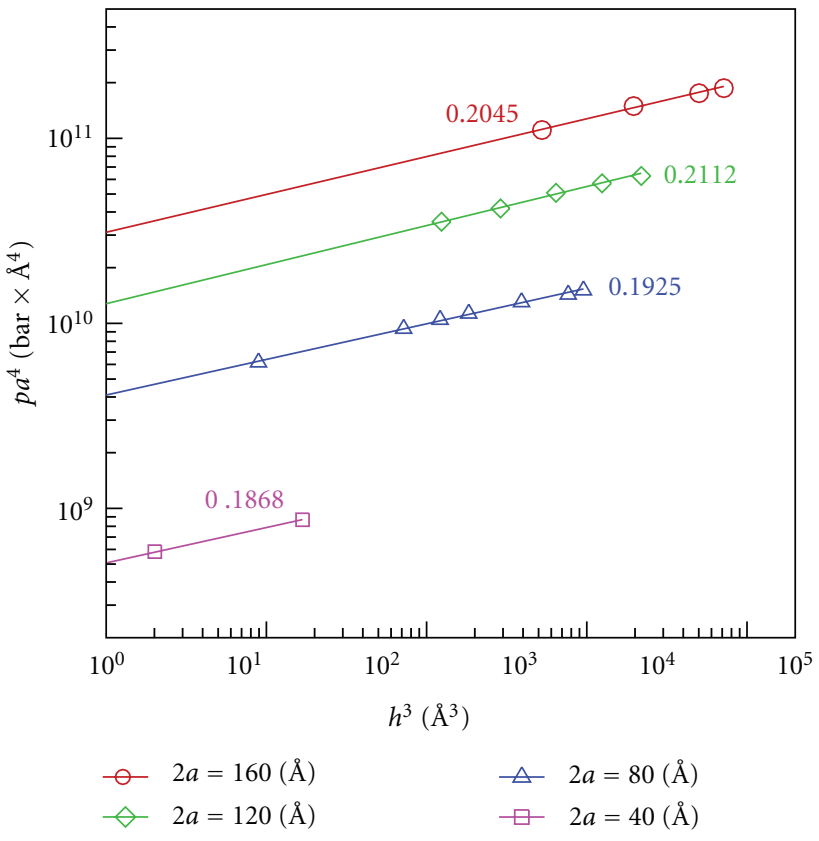

(b)

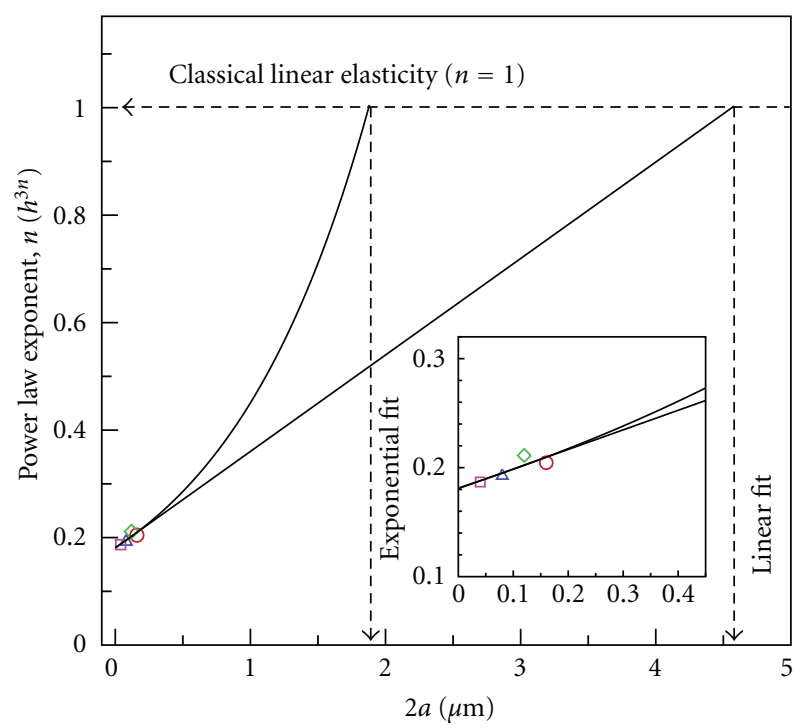

(c)

FIgURE 5: (color online) (a) The relation of $p a^{4}$ and $h^{3}$ follows the power law while linear elastic solution obeys a linear relation. (b) The power law exponents range $n=0.1868 \sim 0.2112$ that are significantly smaller than the linear elastic response of $n=1$. (c) The extrapolations of the estimated power law exponents predict the nonlinear softening behavior to disappear and converge to linear elasticity around the specimen size of $2.0 \sim 4.5 \mu \mathrm{m}$.

height is difficult, particularly when the bulge height (and thus graphene strain) is small. Furthermore, the graphene monolayer does not bulge or strain significantly until a large number of hydrogen molecules are supplied into the chamber. Therefore, at low pressures, the graphene monolayer does not appear to bulge or strain even though the gas pressure is increasing due to the addition of more hydrogen molecules to the chamber, which results in an excessively stiff response from the graphene monolayer until the gas pressure reaches a sufficient value. In order to rule out this difficulty at low gas pressure, we thus collected data only after we were able to capture a noticeably well timeaveraged bulge height at sufficiently high hydrogen pressure. Instead, we simply added a data point of $p=0$ bar at $h=0 \AA$ to our simulation results in order to represent the behavior of graphene under such small pressures (i.e., 
mainly for curve fittings in the vicinity of $h=0 \AA$ as in Figure 3).

We demonstrate the softening behavior and size dependence in two different ways. First, we estimate the nonconstant values of the elastic constant $C_{1}(\varepsilon)$ from the simulation data, using (1), in order to highlight the distinct sizedependent difference from classical linear elasticity (We here drop the higher-order elastic constant $C_{2}$.) Similar estimation can be found in the first-principles calculation of spherical fullerene nanocages filled with hydrogen molecules [11]. Figure 4 shows how the values of the elastic constant calculated from our simulation data decay as the pressure increases. For comparison, the range of the linear elastic constant $(340 \sim 520 \mathrm{~N} / \mathrm{m})$ that is obtained from both the microscale experiments $[2,3]$ and theoretical estimation [12] is also marked on the figure. In Figure 4(a), the four different specimens follow different curves, which demonstrates the size effect. Due to the unrealistically stiff behavior from the low-pressure bulge simulations that was discussed above, we postulate that the four fitted curves will merge into the classical linear elastic value as the bulge height decreases to $h=0 \AA$, or equivalently as the strain in the graphene monolayer due to the hydrogen pressure decreases to zero.

Figure 4(b) demonstrates the size dependence more dramatically. We show this by multiplying the elastic constant $C_{1}$ by the size factor $a^{-3}$, then plotting the product on the $y$-axis of the log scale plot shown in Figure 4(b). Interestingly, all data points from the four different specimen sizes collapse onto a single straight line. Furthermore, the four graphene specimens all exhibit decaying values of the elastic constant as the graphene bulge increases, which again verifies the nonlinear elastic softening behavior of nano-sized graphene monolayers. This softening response to extreme pressure is mainly caused by the stretching and weakening of the carbon-carbon bonds in graphene as explained in the recent first-principles simulation study of fullerene nanocages subjected to extremely high internal pressure [11]. Compared with the fullerene simulation, we obtain lower pressure-to-strain ratios. The reason for this is that in our simulation models, the graphene monolayer covers only a single side of the simulation box, which enables the graphene monolayer to deflect more severely at the same pressure than the fullerene cage that has expandable faces in all directions.

To further demonstrate the softening behavior, we present the plots of $p a^{4}$ versus $h^{3}$ for the four specimens in Figures 5(a) and 5(b). According to (1), the classical linear elastic response is a straight line of which the slope defines the linear elastic constant $C_{1}$ of the graphene monolayer. However, our simulation results are found to be best fitted by power laws, where the power law exponent $n$ in $\left(p a^{4}\right) \sim\left(h^{3}\right)^{n}$ ranges from $n=0.1868 \sim 0.2112$ for the four model specimens as shown in Figure 5(b), while the exponent of classical linear elasticity is $n=1$, that is, $p a^{4} \sim h^{3}$. By extrapolating these four power law exponents, we can approximately predict the graphene monolayer size where this size dependence disappears and begins to follow classical elasticity. In Figure 5(c), we present two possible extrapolations; one is obtained using exponential fitting and the other is obtained using linear fitting. From these results, we can roughly anticipate that the length scale from which classical linear elastic behavior is valid for the pressure bulge test is between about $2.0 \sim 4.5 \mu \mathrm{m}$, which is comparable with the graphene monolayer sizes of about $5 \mu \mathrm{m}$ that were used in the experimental graphene bulge tests [2]. We therefore expect that the nanoscale softening effect will disappear as the size of graphene monolayer reaches the microscale size scales that have been tested experimentally.

\section{Conclusions}

In conclusion, molecular dynamics simulations have been performed to simulate the cylindrical bulge test of nanoscale graphene monolayers under high pressure. The graphene monolayers exhibit size-dependent nonlinear elastic softening as the applied pressure increases due to the stretchinginduced weakening of the carbon-carbon bonds in graphene. Our results demonstrate that the traditional microscale bulge formulas, which assume constant elastic moduli, may need to be modified to incorporate the size dependence and elastic softening for the graphene bulge tests at the nanoscale. Our future research will focus on further investigating the sizedependent softening of graphene through theoretical models that can link the currently presented nanoscale effects to the classical microscale behavior of graphene monolayers along with the effects of temperature.

\section{Acknowledgments}

This work was supported in part by a Faculty Grantin-Aid program from the University of Wyoming. S. Jun acknowledges support from NSF Grant CMMI-0856250. T. Tashi was supported by the University of Wyoming Graduate School and the Government of Tibet in Exile.

\section{References}

[1] J. J. Vlassak and W. D. Nix, "New bulge test technique for the determination of Young's modulus and Poisson's ratio of thin films," Journal of Materials Research, vol. 7, no. 12, pp. 32423249, 1992.

[2] J. S. Bunch, S. S. Verbridge, J. S. Alden et al., "Impermeable atomic membranes from graphene sheets," Nano Letters, vol. 8, no. 8, pp. 2458-2462, 2008.

[3] C. Lee, X. Wei, J. W. Kysar, and J. Hone, "Measurement of the elastic properties and intrinsic strength of monolayer graphene," Science, vol. 321, no. 5887, pp. 385-388, 2008.

[4] I. Szlufarska, "Atomistic simulations of nanoindentation," Materials Today, vol. 9, no. 5, pp. 42-50, 2006.

[5] A. Gouldstone, N. Chollacoop, M. Dao, J. Li, A. M. Minor, and Y.-L. Shen, "Indentation across size scales and disciplines: recent developments in experimentation and modeling," Acta Materialia, vol. 55, no. 12, pp. 4015-4039, 2007.

[6] C. Y. Wang, K. Mylvaganam, and L. C. Zhang, "Wrinkling of monolayer graphene: a study by molecular dynamics and continuum plate theory," Physical Review B, vol. 80, no. 15, Article ID 155445, 2009.

[7] S. Nosé, "A unified formulation of the constant temperature molecular dynamics methods," The Journal of Chemical Physics, vol. 81, no. 1, pp. 511-519, 1984. 
[8] W. G. Hoover, "Canonical dynamics: equilibrium phase-space distributions," Physical Review A, vol. 31, no. 3, pp. 1695-1697, 1985.

[9] D. W. Brenner, O. A. Shenderova, J. A. Harrison, S. J. Stuart, B. Ni, and S. B. Sinnott, "A second-generation reactive empirical bond order (REBO) potential energy expression for hydrocarbons," Journal of Physics Condensed Matter, vol. 14, no. 4, pp. 783-802, 2002.

[10] "LAMMPS Users Manual," http://lammps.sandia.gov/.

[11] O. V. Pupysheva, A. A. Farajian, and B. I. Yakobson, "Fullerene nanocage capacity for hydrogen storage," Nano Letters, vol. 8, no. 3, pp. 767-774, 2008.

[12] F. Liu, P. Ming, and J. Li, "Ab initio calculation of ideal strength and phonon instability of graphene under tension," Physical Review B, vol. 76, no. 6, Article ID 064120, 2007. 

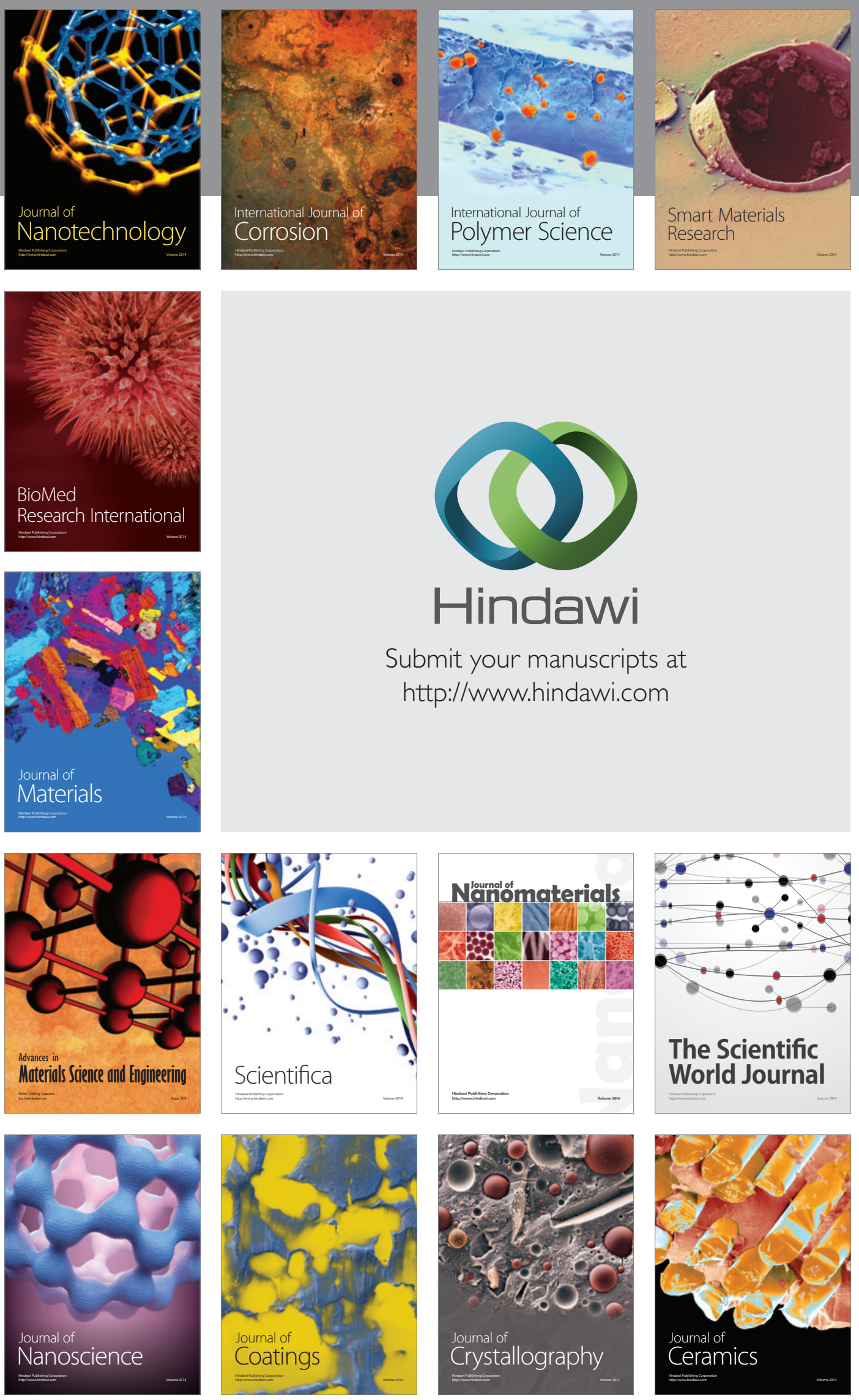

The Scientific World Journal

Submit your manuscripts at

http://www.hindawi.com

\section{World Journal}

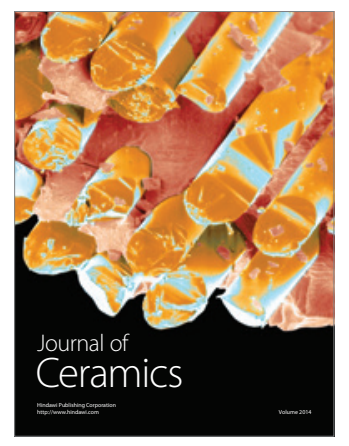

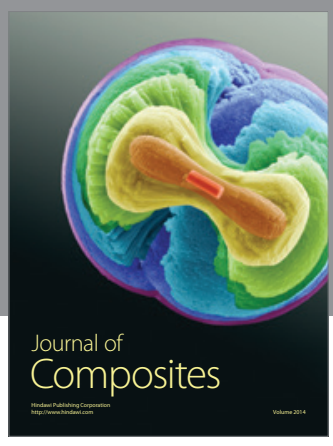
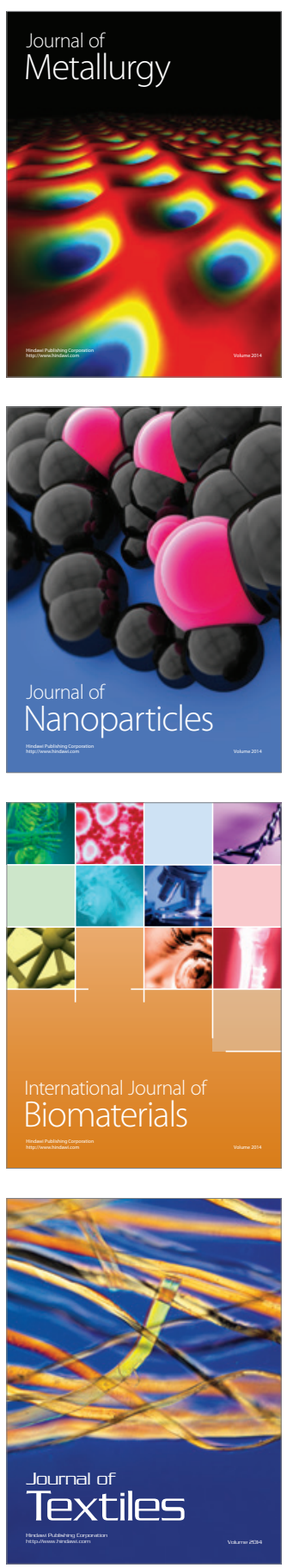